\title{
To Assess the Tuberculosis Situation in Urban and Rural Areas of Bangladesh with Special Emphasis on the Facility of Treatment Scenarios
}

\author{
Sumaiya Rahman ${ }^{1, *}$, Shohel Ahmed ${ }^{2}$ \\ ${ }^{1}$ Department of Statistics, University of Dhaka, Dhaka, Bangladesh \\ ${ }^{2}$ Department of Mathematics, Bangladesh University of Engineering and Technology, Dhaka, Bangladesh
}

\begin{abstract}
Background: Tuberculosis (TB) is one of the major public health challenges in Bangladesh. Though the country has achieved commendable success in Tuberculosis control, yet this success may deem out unless effective TB treatment control measures are taken based on strong general infection control for the diseases over the country. This study aimed to assess facilitators for health seeking practice among the urban and rural peoples Bangladesh. Methods: Present study used secondary data extracted from nationally representative Bangladesh Demographic and Health Survey (BDHS-2011) between May 2, 2014 \& August 14, 2014, on TB patients receiving TB treatments in Urban and Rural areas from Seven Divisions of Bangladesh. Results: Out of 1596 study subjects, 226 TB patients took TB treatment of which Most of the TB patients were from urban $(143,63.3 \%)$, others from Rural $(83,36.7 \%)$ areas. Availability of treatment for TB from government/public health authorities are known to $116(51.3 \%)$ and $82(36.3 \%)$ from urban and rural areas respectively. Our study reflects most of the Tuberculosis medicine facility stored in respective urban service site $(122,54 \%)$ where else no Tuberculosis medicine facility in rural areas is $21(9.3 \%)$. The study has found that, most long term treatment facility has been provided in urban areas compared to rural areas and no association was found between methods to diagnose TB between urban/rural areas. Conclusions: Perceptions of TB and awareness associated with the disease increase the treatment scenarios, therefore promotion of media awareness campaign, engaging the rural people for treatment and effective community service all over the country is needed to increase treatment facility in the future.
\end{abstract}

Keywords Tuberculosis, Urban, Rural, Illness perception, Health seeking practice

\section{Introduction}

Tuberculosis (TB) has been a major public health problem for centuries. It is a leading infectious disease that represents more than a quarter of the world's preventable deaths. Increase in the incidence of TB in the developing countries and its re-emergence in the developed world led the World Health Organisation (WHO) to declare TB as a global emergency in 1993 [1]. Despite the availability of affordable, effective treatment, the annual total of 8.8 million new cases and an estimated 1.6 million of deaths from TB (WHO Report 2007) represents an intolerable burden of human suffering [2].

In Bangladesh, TB is a major public health problem and one of the leading causes of adult mortality and preventable deaths. Tuberculosis (TB) kills an estimated 75,000 people per year in Bangladesh [3]. According to the WHO, among

* Corresponding author:

sumaiyarahman14.sr@gmail.com (Sumaiya Rahman)

Published online at http://journal.sapub.org/phr

Copyright @ 2017 Scientific \& Academic Publishing. All Rights Reserved
22 countries where TB is considered "high burden," Bangladesh is ranked 6th with a mortality rate of $45 / 100,000$ and an incidence rate of 225/100,000 annually, with a prevalence of 411 cases per 100,000 population and 10th among 27 high priority multidrug resistant (MDR) and extensively drug resistant (XDR) TB countries [4].

In Bangladesh, The National TB Control Programme (NTP) coordinates the diagnosis, reporting and treatment of TB cases according to WHO guidelines. In 1993, the Government of Bangladesh started NTP adopting the Directly Observed Treatment Short Course (DOTS) strategy in 4 pilot sub-districts. It was progressively expanded to 460 sub-districts by June 1998. At present the coverage is said to be $99 \%$ including metropolitan cities as Dhaka, Chittagong, Rajshahi, Khulna, Barisal and Sylhet [3]. TB can be completely cured through the Directly Observed Treatment Short Course (DOTS). DOTS is currently practiced as the most effective way of controlling the disease. Even though TB is completely curable, a large number of people still continue to become ill and die from the disease. Many cases remain undiagnosed due to the lack of proper treatment facility. Most TB cases are diagnosed 
through passive case finding. Three sputum specimens are obtained from TB suspects for sputum-smear microscopy. A new smear-positive TB case is diagnosed on the basis of one or more sputum specimens testing positive for acid fast bacilli. Conventional microscopy cannot detect TB bacilli when the concentration is less than 5000 bacilli per $\mathrm{ml}$ of sputum. In Bangladesh, $65 \%$ of the pulmonary TB can be detected by conventional microscopy and the rest $(35 \%)$ remain undiagnosed [5]. These group of TB patients are called smear negative pulmonary TB. Smear-negative TB has to be diagnosed on the basis of symptoms, clinical features, and supportive chest X-ray findings.

A nationally representative survey conducted in 2009 revealed that the prevalence of TB is 10 times higher among the lowest wealth quintile, compared to the highest; and greater in rural areas compared to urban areas. The NTP is credited with implementing directly observed therapy short course (DOTS) countrywide with an impressive 92\% treatment success rate. Though the country has met WHO's case detection rate of $74 \%$, only $14 \%$ of cases are located in urban areas. This calls into question potential barriers and facilitators to both detection and treatment in urban and rural areas among vulnerable populations. The study's results will be helpful for improving the design of TB control activities in urban and rural areas. This study will also provide evidence in the form of feedback to improve the operation of the NTP and toward improving the function of TB control activities in Bangladesh.

\section{Methods}

This study used secondary data extracted from nationally representative Bangladesh Demographic and Health Survey (BDHS-2011) conducted by the authority of the National Institute for Population, Research and Training (NIPORT) of the Ministry of Health and Welfare, Bangladesh and funded by USAID. This study has been carried out between May 2, 2014 \& August 14, 2014, on TB patients receiving TB treatments in urban and rural areas from Seven (Barisal, Chittagong, Dhaka, Khulna, Rajshahi, Rangpur, Sylhet) divisions of Bangladesh.

There were total of 226 study subjects who had under TB treatment in the said duration. Parameters regarding socio-demographic profile include division, urban/rural, type of facility, managing authority.

Study variables include storage of medicine, quality of care system, facility offer for TB tests, treatment for TB etc. Case definitions, treatment outcome definitions and other protocols have been followed as per global tuberculosis report 2016 published by World Health Organization. The data were analysed using IBM SPSS (version 20). Descriptive analysis was done (means, proportions, and percentages) for demographic details. Categorical variables were compared using Chi square test with $\mathrm{P}$ value of $<0.05$ was considered significant.

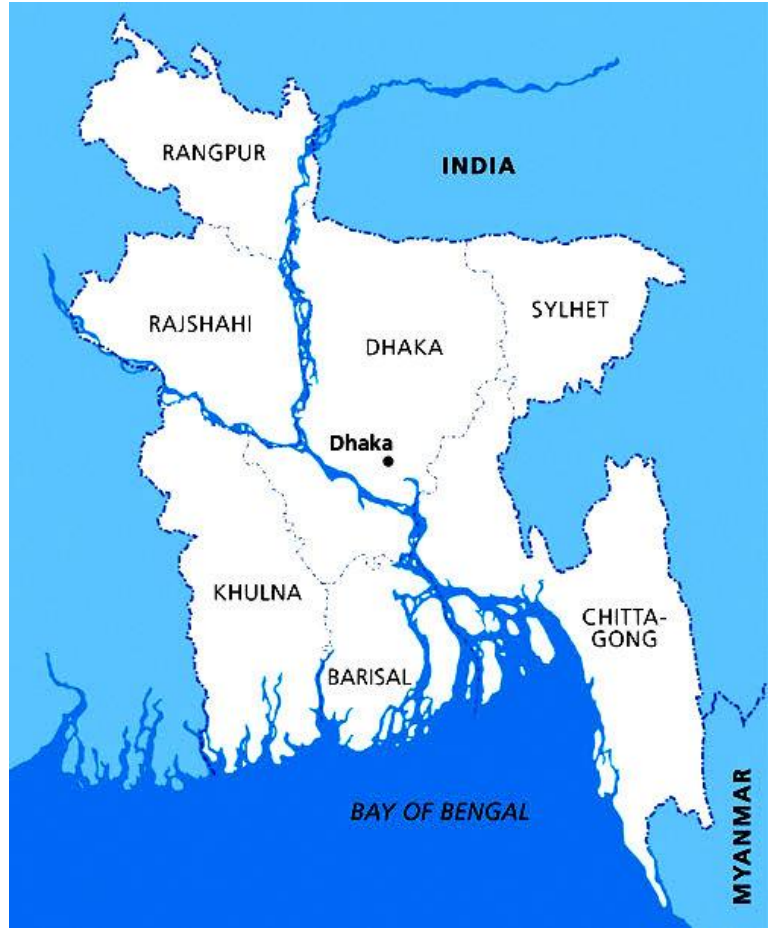

Figure 1. Study subjects from different districts of Bangladesh receiving TB treatment

\section{Results}

A total of 1596 study subjects were included in the final analysis. A total of $226 \mathrm{~TB}$ patients took TB treatment from seven districts of Bangladesh between May, 2014 and August, 2014. Most of the TB patients were from urban (143, $63.3 \%)$, others from rural $(83,36.7 \%)$ areas. Majority of the stakeholders (54 subjects, 23.9\%) were from Dhaka (Capital of Bangladesh), 2nd highest from Chittagong (45 subjects, $19.9 \%)$, followed by $(35,15.5 \%)$ from Barisal, $(25,11.1 \%)$ from Khulna, $(23,10.2 \%)$ from Sylhet, $(22,9.7 \%)$ from Rajshahi and Rangpur (Table 1). Since the data came from BDHS, carried out in 2014, seven divisions were specified in region variable; Mymensingh was not considered separate division in this study since it was declared as new division on 12 January 2015.

Table 1. Distribution of study subjects according to Urban/Rural areas from seven districts of Bangladesh

\begin{tabular}{cccc}
\hline Districts & Urban & Rural & Total(\%) \\
\hline Barisal(\%) & $18(8.0 \%)$ & $17(7.5 \%)$ & $35(15.5 \%)$ \\
Chittagong(\%) & $28(12.4 \%)$ & $17(7.5 \%)$ & $45(19.9 \%)$ \\
Dhaka(\%) & $40(17.7 \%)$ & $14(6.2 \%)$ & $54(23.9 \%)$ \\
Khulna(\%) & $20(8.8 \%)$ & $5(2.2 \%)$ & $25(11.1 \%)$ \\
Rajshahi(\%) & $14(6.2 \%)$ & $8(3.5 \%)$ & $22(9.7 \%)$ \\
Rangpur(\%) & $13(5.8 \%)$ & $9(4.0 \%)$ & $22(9.7 \%)$ \\
Sylhet(\%) & $10(4.4 \%)$ & $13(5.8 \%)$ & $23(10.2 \%)$ \\
\hline Total(\%) & $143(63.3 \%)$ & $83(36.7 \%)$ & $226(100 \%)$ \\
\hline
\end{tabular}


Availability of treatment for TB from government/public health authorities are known to $116(51.3 \%$ ) and $82(36.3 \%)$ from urban and rural areas respectively, where else also local governments $(11,4.9 \%)$, NGO $(9,4.00 \%)$ and private $(7$, $3.1 \%$ ) provide treatment for TB in urban areas. Association between managing authority and urban/rural areas was found statistically significant $(\mathrm{P}=0.002)$ (Table-2). Sputum smear examination is a baseline test for Tuberculosis patients. In our study, it has been found that Sputum smear examination was mostly diagnosed in $76(33.6 \%)$ cases in urban areas and both Sputum and X-ray were diagnosed in 42(18.6\%) and $14(6.2 \%)$ in urban and rural areas respectively. No association was found between method to diagnose TB and urban/rural areas $(\mathrm{P}=0.064)$ (Table-2). Our study reflects most of the Tuberculosis medicine facility stored in respective Urban service site $(122,54 \%)$ where else no
Tuberculosis medicine in rural facility areas is $21(9.3 \%)$. The relationship between medicine storage facility and urban/rural areas was statistically significant $(\mathrm{P}=0.008)$. Our study subjects showed, most long term treatment facility has been provided in urban areas compared to rural areas. The study revealed that, Direct observe 2 months intensive and follow-up 4 Months continuation treatment for Tuberculosis provided in urban areas is $89(39.4 \%)$ cases, followed by Direct observe 6 Month intensive is $30(13.3 \%)$. About $46(20.4 \%)$ and $17(7.5 \%)$ is under 2 months and 6 months intensive treatment in Rural areas respectively. From table-2 it depicts that most of the diagnosed prescribed meds, routine and follow-up treatments provided by different clinics/units is urban areas. The study has found that, the association between treatment for $\mathrm{TB}$ and urban/rural areas was statistically insignificant $(\mathrm{P}=0.177)$ (Table-2).

Table 2. Association of Tuberculosis treatment and facility scenarios with Urban and Rural areas

\begin{tabular}{|c|c|c|c|}
\hline Characteristic & $\operatorname{Urban}(\%)$ & Rural(\%) & P Value \\
\hline \multicolumn{4}{|l|}{ Managing Authority } \\
\hline Government/Public & $116(51.3 \%)$ & $82(36.3 \%)$ & \\
\hline Local Government & $11(4.9 \%)$ & $0(0 \%)$ & \\
\hline NGO & $9(4.0 \%)$ & $1(0.4 \%)$ & \\
\hline Private & $7(3.1 \%)$ & $0(\%)$ & $0.002 *$ \\
\hline \multicolumn{4}{|l|}{ Method to diagnose TB } \\
\hline No TB diagnosis services & $1(0.4 \%)$ & $7(3.1 \%)$ & \\
\hline Sputum smear only & $76(33.6 \%)$ & $28(12.4 \%)$ & \\
\hline $\mathrm{X}$-ray only & $1(0.4 \%)$ & $1(0.4 \%)$ & \\
\hline Either sputum or $\mathrm{x}$-ray & $11(4.9 \%)$ & $4(1.8 \%)$ & \\
\hline Both sputum and x-ray & $42(18.6 \%)$ & $14(6.2 \%)$ & \\
\hline Clinical symptoms only & $8(3.5 \%)$ & $13(5.8 \%)$ & \\
\hline Refer to outside facility & $4(1.8 \%)$ & $16(7.1 \%)$ & 0.064 \\
\hline \multicolumn{4}{|l|}{ Medicine storage facility } \\
\hline No TB meds in facility area & $14(6.2 \%)$ & $21(9.3 \%)$ & \\
\hline Stored in service site & $122(54.0 \%)$ & $58(25.7 \%)$ & \\
\hline Stored in pharmacy & $7(3.1 \%)$ & $4(1.8 \%)$ & $0.008^{*}$ \\
\hline \multicolumn{4}{|l|}{ Treatment for TB } \\
\hline Direct observe 2 Month intensive, Follow-Up 4 Month continuation & $89(39.4 \%)$ & $46(20.4 \%)$ & \\
\hline Direct observe 6 Month intensive & $30(13.3 \%)$ & $17(7.5 \%)$ & \\
\hline Follow up clients only after first 2 Month intensive direct observation elsewhere & $3(1.3 \%)$ & $1(0.4 \%)$ & \\
\hline Diagnose and treat while inpatient. Discharge to other clinic/unit for follow up & $3(1.3 \%)$ & $1(0.4 \%)$ & \\
\hline Provide full treat with no routine direct observation phase & $2(0.9 \%)$ & $0(0.0 \%)$ & \\
\hline Diagnose, prescribe/provide meds, no follow up & $9(4.0 \%)$ & $5(2.2 \%)$ & \\
\hline Diagnose only, no treat or prescription of medications & $7(3.1 \%)$ & $13(5.8 \%)$ & 0.177 \\
\hline
\end{tabular}

*Statistically significant $(\mathrm{p}<0.05)$. 


\section{Discussion}

In the present study it was found that $226 \mathrm{~TB}$ patients took TB treatment from seven districts of Bangladesh of which most of the TB patients were from urban $(143,63.3 \%)$, others from Rural $(83,36.7 \%)$ areas. A study conducted by Sayera Banu et al. on Tuberculosis in an urban Slum of Dhaka City revealed high prevalence of TB in an urban slum area which is more than two times higher than overall prevalence and nearly four times higher than the prevalence in urban settings [6]. Another study by Hossain, S showed in $2007,18.5 \%$ of all TB cases were reported from urban areas that make up $28 \%$ of the country's population. This poor case finding in urban areas might happened due to delayed and lower coverage of DOTS in the urban areas and presence of excessive numbers of practitioners not connected to the NTP [7]. A study on Control of tuberculosis by community health workers in Bangladesh by Mushtaque R Chowdhury et al. showed that tuberculosis is more common in urban than rural areas [8].

Our study showed that the availability of treatment for TB from government/public health authorities is known to (198, $87.9 \%)$, where else private sector provides $(7,3.1 \%)$. A study on government-NGO collaboration in the case of tuberculosis control in Bangladesh by Zafar Ullah et al. showed that most TB patients prefer to seek care from the private sectors. This study also revealed the lowest-level health facilities providing appropriate TB care are Upazila Health Complexes located in the upazila headquarters (one hospital per 250000 population). To improve reproductive health services GOB implement DOTS in Upazilas and four metropolitan cities with the collaboration of different NGOs [9].

In the present study most of the Tuberculosis long term treatment facility and medicine stored has been provided in urban areas. Study conducted by Ziaul Islam et al. on case detection of tuberculosis among children in Bangladesh showed supply and maintenance of necessary diagnostics and child friendly TB drugs remained suboptimal in the country [10]. In 2014 Child TB case detection was $97 \%$ in urban and 13\% in rural areas. A study on 432 key community members, $229(53 \%)$ of all had good knowledge regarding TB and only 13 (3\%) of them had poor knowledge [11]. A results from a countrywide care seeking tuberculosis survey in Bangladesh provided that a total of 273 TB cases, 240 (88\%) from the TB registers. Among the 240 cases detected passively under NTP, $118(49.1 \%)$ sought initial care from informal providers, $106(44.2 \%)$ from formal providers and $16(6.7 \%)$ used self-care [11].

This study revealed several key findings that will help to strengthen future TB control efforts in urban and rural areas in Bangladesh. We observed that the facility of treatment was altogether different in urban and rural areas of Bangladesh with respect to prescribing treatment and availability of drugs. However, unlike in the urban areas, there was almost a complete absence of diagnostic procedures and referrals in the rural areas. It can be said that most of the treatment facility for TB cases was sought from public sectors and limited to urban areas. A large proportion of patients remained in the rural areas during subsequent infection. It is imperative that the NTP should immediately take the initiative to engage all types of care providers, particularly the private sectors and NGOs. It is also necessary to strengthen the on-going advocacy communication and social mobilization activities to increase awareness of key TB symptoms, availability of diagnosis and medicine in an attempt to prevent Tuberculosis in Bangladesh.

\section{Conclusions}

Tuberculosis long term treatment facility services are still underutilized and informal caregivers remained the major care providers for such cases in Bangladesh. In order to improve treatment strategy, it is necessary that the National Tuberculosis Programme immediately takes effective initiatives to engage all types of care providers including public and private sectors, particularly community service providers who are the first point of care for the majority of the TB suspects. Current TB treatments have proven to be more successful to facilitate urban people, while underprivileged populations lived in rural areas have fallen through the gaps in the system. So the NTP should implement strategies to raise community awareness alongside increasing the capacity of service providers and ensuring availability of diagnostics and pediatric TB drugs at the rural level.

\section{ACKNOWLEDGEMENTS}

The authors wish to thank The Demographic and Health Surveys (http://dhsprogram.com/) [12] and Bangladesh Demographic and Health Survey for providing primary data used in this study. The authors would specifically like to thank NIPORT and USAID for their assistance in conducting Bangladesh Demographic and Health Survey (BDHS-2014).

\section{REFERENCES}

[1] World Health Organization. Global tuberculosis report 2016.

[2] WHO (2016) World health statistics 2016: monitoring health for the SDGs, sustainable development goals.

[3] National Guidelines for Tuberculosis Infection Control, National Tuberculosis Control Programme, Directorate General of Health Services Ministry of Health and Family Welfare Dhaka, Bangladesh.

[4] Tuberculosis Control in Bangladesh: Annual Report 2016, National Tuberculosis Control Program Directorate General of Health Services Mohakhali, Dhaka-1212.

[5] National Guidelines for the Management of Tuberculosis in 
Children, National Tuberculosis Control Programme, Directorate General of Health Services Ministry of Health and Family Welfare Dhaka, Bangladesh.

[6] Banu Sayera, Rahman Toufiq, Khatun Razia, et al. Epidemiology of Tuberculosis in an Urban Slum of Dhaka City, Bangladesh. PLoS One. 2013 Oct 21; 8(10).

[7] Hossain, S. Tuberculosis burden in Bangladesh: epidemiological estimates and people's perspectives. PhD thesis (2014), Faculty of Medicine (AMC-UvA), University of Amsterdam, Secretariat, Singel 425, 1012 WP Amsterdam, The Netherlands. Available at http://dare.uva.nl/search?ident ifier $=$ b9506961-45a5-4de0-ad4d-c134d3e2bb28. Accessed 9 April 2017.

[8] Chowdhury A M R, Chowdhury Sadia, et al. Control of tuberculosis by community health workers in Bangladesh. THE LANCET. 19 July 1997; Volume 350, No. 9072: 169-172.
[9] Zafar Ullah AN, Newell JN, Ahmed JU, Hyder MK, Islam A. Government-NGO collaboration: the case of tuberculosis control in Bangladesh. Health Policy Plan. 2006 Mar; 21(2): 143-55.

[10] Islam Ziaul, Istiaque Sanin Kazi, Ahmed Tahmeed. Improving case detection of tuberculosis among children in Bangladesh: lessons learned through an implementation research. BMC Public Health. 2017; 17: 131.

[11] Hossain S, Zaman K, Quaiyum A, et al. Care seeking in tuberculosis: results from a countrywide cluster randomized survey in Bangladesh. BMJ Open 2014; 4:e004766. doi:10.1136/bmjopen-2013-004766.

[12] Demographic and Health Surveys (http://dhsprogram.com/). 